\title{
Detailed balance calculations for hot-carrier solar cells: coupling high absorptivity with low thermalization through light trapping
}

\author{
Maxime Giteau ${ }^{1,2, *}$, Daniel Suchet ${ }^{1,3}$, Stéphane Collin ${ }^{1,4}$, Jean-François Guillemoles ${ }^{1,3}$, and Yoshitaka Okada ${ }^{1,2}$ \\ ${ }^{1}$ NextPV, LIA RCAST-CNRS, The University of Tokyo, Komaba 4-6-1, Meguro-ku, Tokyo 153-8904, Japan \\ 2 Research Center for Advanced Science and Technology, The University of Tokyo, Komaba 4-6-1, Meguro-ku, Tokyo 153-8904, \\ Japan \\ ${ }^{3}$ CNRS, Institut Photovoltaique d'Ile de France (IPVF), UMR 9006, 30 route départementale 128, 91120 Palaiseau, France \\ ${ }^{4}$ Centre for Nanoscience and Nanotechnology (C2N), CNRS, University Paris-Sud/Paris-Saclay, Route de Nozay, \\ 91460 Marcoussis, France
}

Received: 13 November 2018 / Received in final form: 13 March 2019 / Accepted: 19 April 2019

\begin{abstract}
Hot-carrier solar cells could enable an efficiency gain compared to conventional cells, provided that a high current can be achieved, together with a hot-carrier population. Because the thermalization rate is proportional to the volume of the absorber, a fundamental requirement is to maximize the density of carriers generated per volume unit. In this work, we focus on the crucial role of light trapping to meet this objective. Using a detailed balance model taking into account losses through a thermalization factor, we obtained parameters of the hot-carrier population generated under continuous illumination. Different absorptions corresponding to different light path enhancements were compared. Results are presented for open-circuit voltage, at maximum power point and as a function of the applied voltage. The relation between the parameters of the cell (thermalization rate and absorptivity) and its characteristics (temperature, chemical potential, and efficiency) is explained. In particular, we clarify the link between absorbed light intensity and chemical potential. Overall, the results give quantitative values for the thermalization coefficient to be achieved and show that in the hot-carrier regime, absorptivity enhancement leads to an important increase in the carrier temperature and efficiency.
\end{abstract}

Keywords: Hot-carrier solar cells / detailed balance / ultrathin absorber / light trapping / thermalization / chemical potential

\section{Introduction}

Hot-carrier solar cells (HCSCs) promise efficiencies way above the single junction limits [1], but face many challenges, one of them being the establishment of a hotcarrier population under continuous illumination. In conventional cells under reasonable concentration, the extra kinetic energy of the absorbed photons is lost through scattering with phonons in a process called thermalization. In order to reach a hot-carrier regime, one must increase the intensity of absorbed light and/or decrease the thermalization rate. Because the latter scales with the volume of the absorber [2], HCSC fundamentally requires to maximize the density of carriers generated per unit of volume [3].

So far, most efforts have been put into the development of quantum structures [4] in order to reduce the thermalization. Relatively low thermalization coefficients have been reported [5], along with strong evidence of

\footnotetext{
* e-mail: maximegiteau@hotmail.fr
}

hot-carrier current extraction [6,7]. We will show that current state-of-the-art thermalization coefficients could be suitable for an increase in the efficiency of solar cells beyond the Shockley-Queisser limit. However, those results were obtained for a single wavelength laser illumination, and with very thin absorbers with poor broadband absorptivity, so that they cannot work efficiently as solar cells. It is, therefore, necessary to work not only on the reduction of the thermalization coefficient but also on maintaining high broadband absorptivity.

In this context, we investigated the influence of light trapping on the temperature, chemical potential, and efficiency of solar cells. The objective of this paper is to show that advanced light trapping is a necessity. Even more, it is the missing part needed in order to demonstrate the potential interest of HCSC.

We develop the theoretical framework and algorithm for detailed balance calculations adapted to HCSC in the radiative limit. It takes into account the absorptivity of the material. This is the simplest model that combines the parameters of which we want to see the influence. Light 
trapping is introduced in the form of uniform broadband light path enhancement that increases the effective thickness of the material for all wavelengths.

The paper is organized into three sections. In the first section, we explain the model, from the calculation of the absorption and the detailed balance to the $J(V)$ characteristic. Then we present the results of the influence of light trapping and the thermalization coefficient at open circuit, maximum power point, and when sweeping the voltage. Finally, we discuss the obtained results based on reference models and existing literature.

\section{Methods}

\subsection{Absorptivity: band filling and light path enhancement}

We aim at investigating the influence of absorption enhancement on HCSCs. We introduce a dimensionless light path enhancement factor $F$ such that the actual distance traveled by light in the material is longer than its actual thickness by a factor $F$. The absorptivity of a material follows the Beer-Lambert law, and assuming a perfect anti-reflection coating, we obtain:

$$
A=(1-\exp (-F \alpha d))
$$

where $\alpha$ is the absorption coefficient and $d$ is the thickness of the absorber. Several cases can be identified for the light path enhancement. $F=2$ is the double-pass absorption obtained with a perfect back mirror, $F=4 n^{2} \approx 50$ is the Lambertian case [8], while $F=4 \pi n^{2} \approx 150$ is the upper bound value for multiresonant light trapping [9].

The absorption coefficient in equation (1) is band-filling dependent, meaning that the more electrons are occupying a given energy level in the conduction band (and the fewer in the valence band), the less likely it is for the material to absorb photons. We can link $\alpha$ to another absorption coefficient $\alpha_{0}$, obtained in absence of band-filling (for low incident photon flux), writing:

$$
\alpha(E)=\alpha_{0}(E)\left[1-f_{h}\left(E_{h}\right)-f_{e}\left(E_{e}\right)\right]
$$

with $f_{e}\left(E_{e}\right)$ and $f_{h}\left(E_{h}\right)$ the Fermi-Dirac distributions for electrons in the conduction band and holes in the valence band, respectively. We suppose that the electron and hole populations are independent, each in thermodynamic equilibrium with their own Fermi energy $E_{F, e}$ and $E_{F, h}$, and at the same temperature $T$, which does not have to be the temperature of the lattice. In these conditions the distributions can be written:

$$
\begin{aligned}
f_{e}\left(E_{e}\right) & =\frac{1}{1+\exp \left(\frac{E_{e}-E_{F, e}}{k_{B} T}\right)} \\
f_{h}\left(E_{h}\right) & =\frac{1}{1+\exp \left(\frac{E_{F, h}-E_{h}}{k_{B} T}\right)} .
\end{aligned}
$$

For the sake of simplicity, we further assume that the dispersion relation of electrons and holes can be written using an effective mass and that this mass is the same for electrons and holes. The same calculation could be performed with different hole and electron temperatures and/or effective masses [10]. Also, it has been experimentally confirmed that in polar semiconductors, electrons (with a much lower effective mass) heat much more than holes [11]. However, considering different electron and hole behaviors makes the interpretation of the results much more difficult, while it does not bring any further insight into this study.

We can show that under those assumptions (same effective mass and same temperature for both carriers), the band-filling dependence takes the form [7]:

$$
\alpha(E)=\alpha_{0}(E) \tanh \left(\frac{E-\mu}{4 k_{B} T}\right)
$$

where $k_{B}$ is the Boltzmann constant and $\mu$ is the difference between the chemical potentials of holes and electrons:

$$
\mu=E_{F, e}-E_{F, h} .
$$

\subsection{Detailed balance}

We consider the radiative limit case, where all absorbed photons lead to an electron-hole pair which is either collected or recombines radiatively (by re-emitting a photon). The detailed balance, developed by Shockley and Queisser in 1961 [12], states that at thermodynamic equilibrium:

$$
J_{\text {ext }}=J_{\text {abs }}-J_{\text {rad }}
$$

Light arrives from the sun and a current density $J_{\text {abs }}$ is absorbed. The cell radiates light with a total flux density $J_{\text {rad }}$. The net current $J_{\text {ext }}$ is collected through the electrical contacts. In the case of conventional cells, the resolution of this equation enables us to obtain the $J_{\text {ext }}(V)$ characteristic.

Kirchhoff law of thermal radiation under thermodynamic equilibrium states that for any energy $E$ and solid angle $\Omega$, the emissivity $\varepsilon$ is equal to the absorptivity $A$ :

$$
A(E, \Omega)=\varepsilon(E, \Omega)
$$

Assuming an isotropic emission in the half-space above the cell (we suppose a mirror keeps the photons from escaping from the back), the radiated current density can be expressed as:

$$
J_{\mathrm{rad}}=q \pi \int A(E) \phi(E) d E
$$

where $q$ is the electron charge and $\phi(E)$ is the spectral photon flux. This flux, in $\mathrm{W}^{-1} \mathrm{~m}^{-2} \mathrm{sr}^{-1}$, is obtained from the generalized Planck's law [13]:

$$
\phi(E)=\frac{2 E^{2}}{h^{3} c^{2}\left(\exp \left(\frac{E-\mu}{k_{B} T}\right)-1\right)}
$$


where $h$ is the Planck constant and $c$ is the speed of light in vacuum. Under the hypotheses of identical electron and hole temperature and effective mass, $T$ is both the temperature of the carriers and of the radiation, and the chemical potential $\mu$ of the radiation is the separation of the Fermi levels (Eq. (6)) [7].

The input current $J_{\text {abs }}$ can be written in a similar way as equation (9), by modifying the expression of the photon flux and the angular integration. We consider the AM1.5G solar spectrum. Its total irradiance for one sun is $100 \mathrm{~mW} \mathrm{~cm}^{-2}$, and we consider full light concentration $(C=45000)$.

In the case of HCSC, the radiation temperature is different from the lattice temperature: the extra kinetic energy the carriers receive when absorbing a photon does not fully thermalize, hence a new steady-state configuration is reached, and a new equilibrium relation on the power is required. It was first studied by Ross and Nozik [1], and leads to the system [14]:

$$
\left\{\begin{array}{l}
J_{\text {ext }}=J_{\text {abs }}-J_{\text {rad }} \\
P_{\text {ext }}=P_{\text {abs }}-P_{\text {rad }}-P_{\text {th }} .
\end{array}\right.
$$

$P_{\text {abs }}$ and $P_{\text {rad }}$ take the same form as $J_{\text {abs }}$ and $J_{\text {rad }}$, by multiplying the photon flux by the photon energy $E$, changing it from a particle to an energy flux. $P_{\text {th }}$ is the thermalized power, and $P_{\text {ext }}$ is the power that is taken out of the absorber.

\subsection{Thermalization}

The main source of energy loss for hot-carriers in a polar semiconductor (typically GaAs) is the emission of longitudinal optical (LO) phonons [15]. If we assume they are the only source of thermalization, the thermalized power $P_{\text {th }}$ can be written [2]:

$$
P_{\text {th }}=Q\left(T-T_{L}\right) \exp \left(-\frac{E_{\mathrm{LO}}}{k_{B} T}\right)
$$

where $T_{L}$ is the lattice temperature, $E_{\mathrm{LO}}$ is the energy of the LO phonon mode, and $Q$ is the so-called thermalization factor in $\mathrm{W} \mathrm{cm}^{-2} \mathrm{~K}^{-1}$, which only depends on the material characteristics. Therefore, this thermalized power is only dependent on the carrier temperature. This law is highly empirical, and the thermalization factor is a parameter which is determined experimentally [2]. As such, we will be using this factor as an adjustable parameter to help us identify which range of values could be suitable for hot-carrier absorbers.

By considering a single thermalization coefficient, we implicitly assume that the electron-phonon interaction is identical to the hole-phonon interaction, which is necessary for assuming identical electron and hole temperatures.

\subsection{Resolution for open circuit condition and maximum power output}

\subsubsection{Open circuit}

At open circuit, there is not current or power extraction, so the system to be solved is reduced to:

$$
\left\{\begin{array}{l}
J_{\mathrm{abs}}=J_{\mathrm{rad}} \\
P_{\mathrm{abs}}=P_{\mathrm{rad}}+P_{\mathrm{th}} .
\end{array}\right.
$$

This 2-equation system is a function of two parameters: $\mu$ and $T$, and we suppose it admits a single solution. The rigorous demonstration of the unicity of this solution is beyond the scope of this work, but it was shown to be true at least in Boltzmann approximation [16].

\subsubsection{Maximum power point}

To obtain the maximum efficiency of the cell, one needs to find the bias that results in the highest power output. Thus, one has to relate the voltage $V$ of the cell to the chemical potential of the radiation. This can be done using selective contacts with a given width and extraction energy. For simplicity here, we will suppose the extraction is isentropic (in reality, some entropy must be generated during extraction [17]). By equilibrating the populations in the absorber and in the contacts, it results in [1]:

$$
q V=\frac{T_{L}}{T} \mu+\left(1-\frac{T_{L}}{T}\right) E_{\text {ext }} .
$$

The extracted current and power are related through the extracted energy $E_{\text {ext }}$ :

$$
P_{\text {ext }}=E_{\text {ext }} J_{\text {ext }}
$$

This extracted power is different from the power output of the cell. It describes how much energy is removed from the absorber, in the same way that $E_{G} \times J_{\text {ext }}$ is the power removed from the absorber in a conventional cell. The output power $P$ of the system is expressed as a function of the voltage:

$$
P=V J_{\mathrm{ext}}=\frac{T_{L}}{T} \mu J_{\mathrm{ext}}+\left(1-\frac{T_{L}}{T}\right) P_{\mathrm{ext}} .
$$

Equation (16) can be maximized as a function of the two parameters $\mu$ and $T$ to give the overall maximum efficiency. This maximum efficiency corresponds to a given $E_{\text {ext }}$, obtained from equation (15), which is the extraction energy that will result in the highest efficiency at the maximum power point operation.

\subsubsection{J(V) curve}

Finally, to obtain the $J(V)$ characteristic and other properties as a function of the voltage, we fix the extraction energy to its value at the maximum power point, and we solve the system of equations (14) and (15) for a given voltage $V$ from 0 to $V_{\text {OC }}$.

\subsection{Case considered}

Because we are working in the radiative limit case, we consider an intrinsic absorber of GaAs, a material whose radiative efficiency can be one of the closest to the ideal case [18]. The absence of intentional doping results in identical electron and hole concentrations under illumination, where the intrinsic doping can be neglected. We consider the absorption coefficient $\alpha_{0}$ as obtained from Palik [19], modified with a parabolic fitting near the bandgap. We 


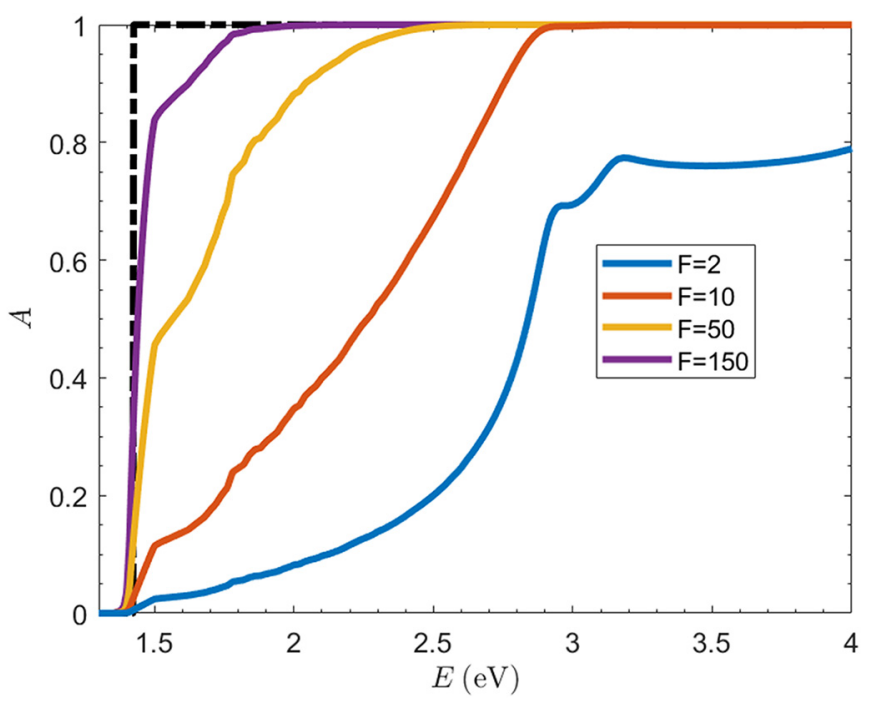

Fig. 1. Absorptivity as a function of the photon energy in $10 \mathrm{~nm}-$ thick GaAs for the 4 cases considered, with various light path enhancement factors $F$. The step absorber is shown for reference (dashed black).

then add the band filling (Eq. (5)) to obtain the absorption coefficient $\alpha$.

The thickness $d$ considered in equation (1) is fixed to $10 \mathrm{~nm}$. At such small scales, the energy states start being quantized, which is even more sensible for hot-carrier temperatures, as the population is more spread in energy. Because the objective here is to make a comparison between weakly and highly absorbing materials, we will still consider the absorption coefficient to be the same as bulk. The cases to be compared are $F=2$ (double pass absorption: perfect mirror), $F=10, F=50$ (Lambertian scattering), and $F=150$ (multi-resonant upper bound). We show in Figure 1 the absorptivity in each case, in the absence of band filling. We see that $F=150$ is very close to the step absorber limit of GaAs, and we confirmed that the results are very similar with both (see Supplementary Fig. S1).

\section{Results}

\subsection{Open circuit}

First, we solve the system (13) for each case, and with a varying thermalization coefficient, to obtain the chemical potential $\mu$ and temperature $T$ of the carriers. The results are presented in Figure 2. The right part of the figure, for a high thermalization coefficient, tends toward a fully thermalized absorber $\left(T=T_{L}, \mu=V_{\mathrm{OC}}\right)$, while the left part tends toward the ideal HCSC (no thermalization or $Q=0$ ). We observe that when decreasing the thermalization coefficient, the temperature increases while the chemical potential decreases. Moreover, we observe that for an increasing value of the light trapping, we have always a higher temperature and a lower chemical potential. More specifically, when increasing the light path enhancement, the switch between conventional cell and ideal HCSC is sharper, occurs at a higher thermalization factor (approximately from $Q=1 \mathrm{~W} \mathrm{~cm}^{-2} \mathrm{~K}^{-1}$ for $F=2$ to $Q=10 \mathrm{~W} \mathrm{~cm}^{-2} \mathrm{~K}^{-1}$ for

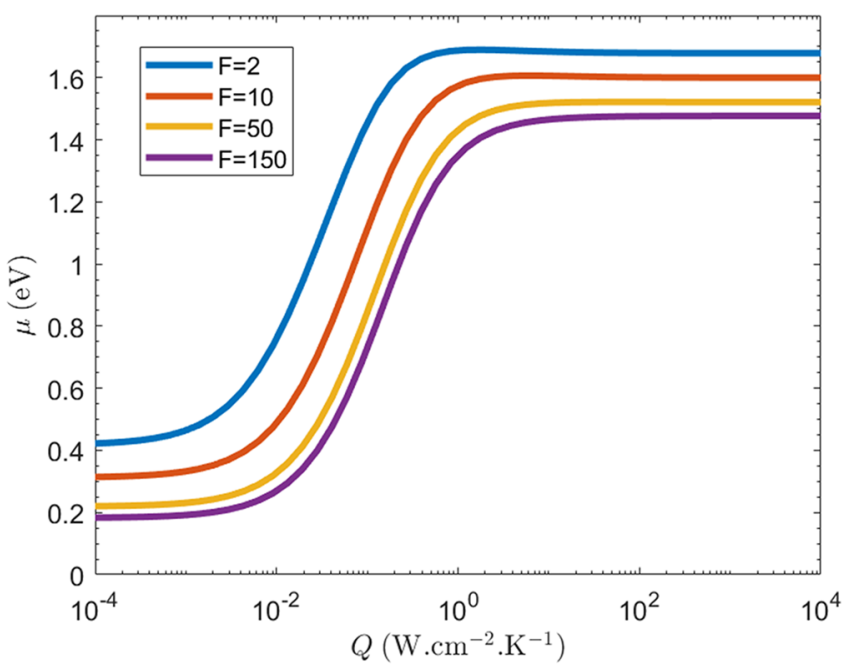

(a)

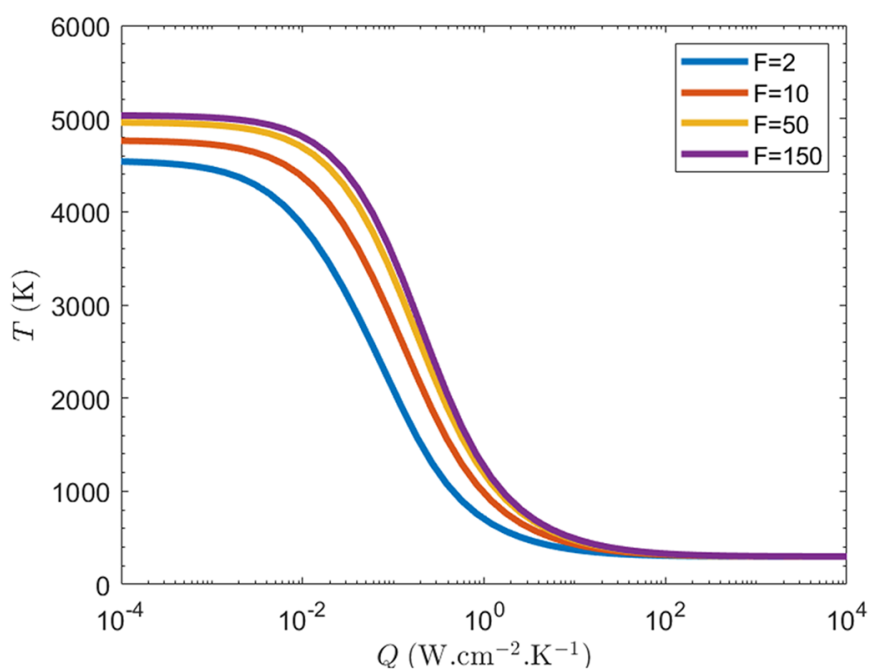

(b)

Fig. 2. (a) Chemical potential and (b) temperature of the carriers in open-circuit condition as a function of the thermalization coefficient $Q$, for different absorption cases.

$F=150$ ), and the temperature reached at zero thermalization is higher (from 4600 to $5000 \mathrm{~K}$ ).

\subsection{Maximum power point}

We also solve the problem at maximum power point using equation (16), and obtain in addition of the chemical potential and the temperature, the maximum efficiency attainable for a given thermalization coefficient (Fig. 3). We still verify that the temperature increases and the chemical potential decreases with decreasing thermalization coefficient. We observe that except for very low thermalization, higher absorptivity still leads to a higher temperature and lower chemical potential (as in open circuit). Also, in this case, the chemical potential can become negative for very low thermalization. Overall, the efficiency, shown in Figure 3c, exhibits an increase with increasing light trapping and decreasing thermalization. For $Q \gg 1 \mathrm{~W} \mathrm{~cm}^{-2} \mathrm{~K}^{-1}$, the efficiency is the same as for a conventional cell in the radiative limit. 


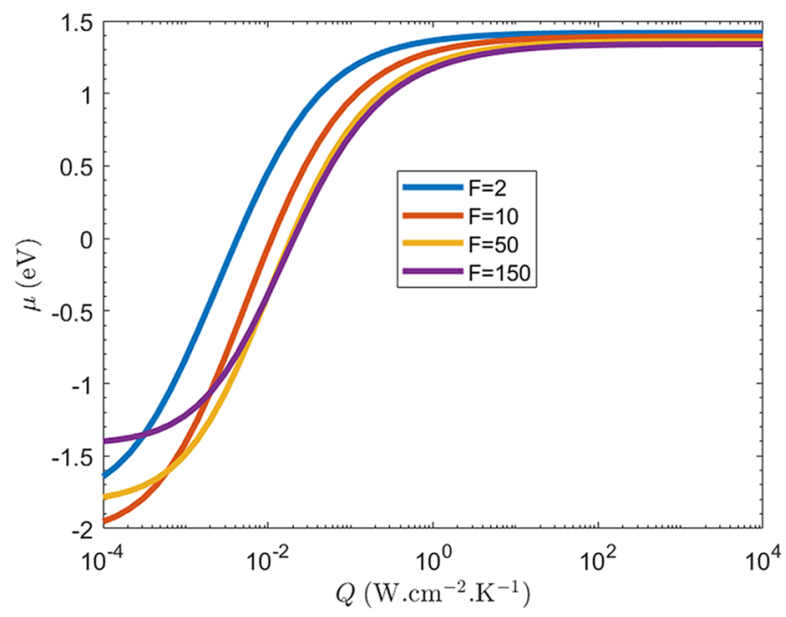

(a)

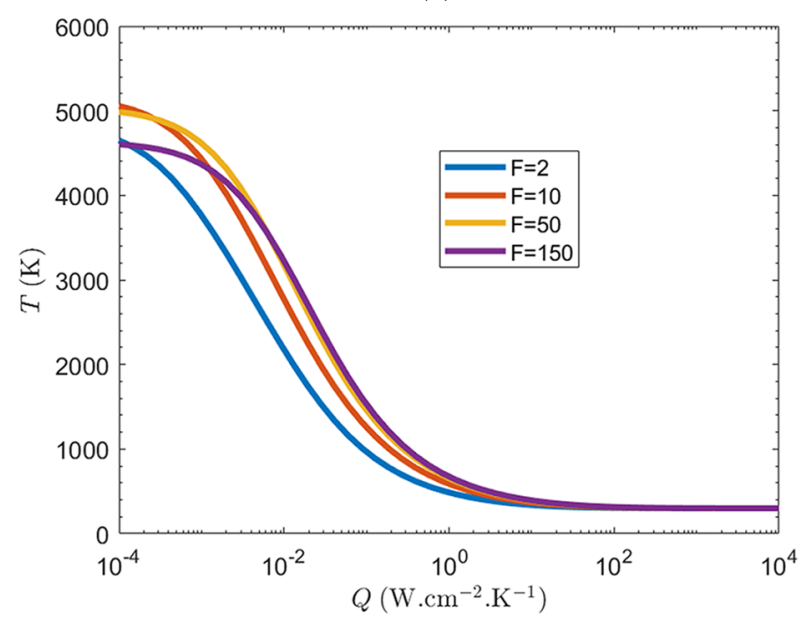

(b)

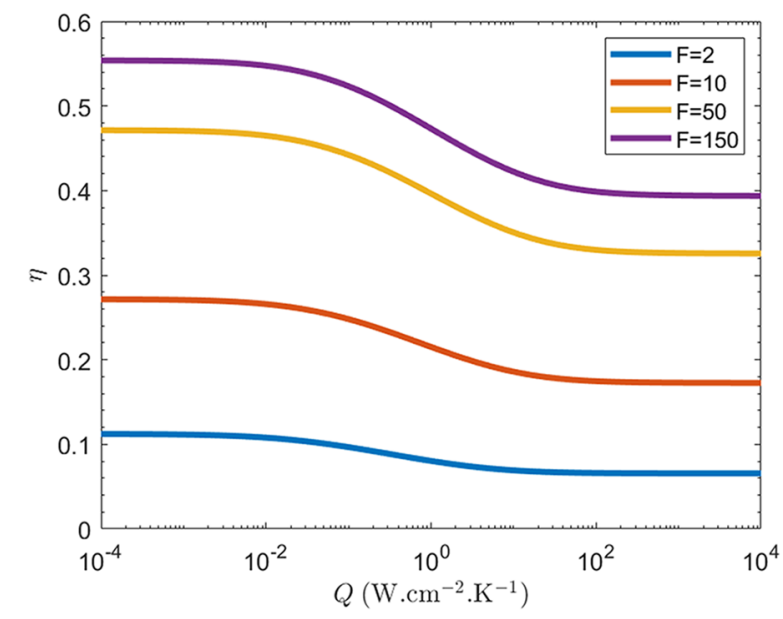

(c)

Fig. 3. (a) Chemical potential, (b) temperature of the carriers, and (c) efficiency at the maximum power point, as a function of the thermalization coefficient $Q$, for different absorption cases.

\section{$3.3 J(V)$ curve}

Finally, we present the evolution of the parameters of the HCSC as a function of the voltage $V$, with $E_{\text {ext }}$ fixed from the maximum power point value, in Figure 4. The extracted current $J_{\text {ext }}$ is divided by the concentration (45000 suns), to display a value per sun. We also present the different parameters obtained for conventional solar cell and HCSC in Supplementary Table S1. They are calculated for $Q \gg 1 \mathrm{~W} \mathrm{~cm}^{-2} \mathrm{~K}^{-1}$.

We can first observe from Figure 4c that the behavior of conventional cells is very similar to HCSC. In particular, their $J_{\mathrm{sc}}$ is the same, and they seem to follow the same slope near $V_{\mathrm{OC}}$, except that the value of the latter is higher for HCSC. The FF is also very similar, so that an efficiency enhancement of about $20 \%$, mainly due to a higher voltage, is observed. The efficiency also increases with increasing light path enhancement. A strong gain occurs in the $J_{\mathrm{SC}}$, and the FF is also improved, which largely compensates for a decrease in $V_{\text {OC }}$.

We also show in Figures $4 \mathrm{a}$ and $4 \mathrm{~b}$ the corresponding chemical potential and temperature, respectively. We plot them for voltages slightly above $V_{\mathrm{OC}}$ (where the current is negative), in order to observe the general trend. The chemical potential is first strongly negative and increases linearly up to $V_{\mathrm{mpp}}$, after which it still increases but much more slowly. The temperature is first constant (at a temperature higher than $T_{L}$ ), before increasing sharply after $V_{\text {mpp }}$. Also, the temperature is systematically higher with light trapping, and the chemical potential increases with a variable slope. For $V=0$ and $V_{\mathrm{OC}}$, the chemical potential is lower with higher light path enhancement, but the curves happen to intersect around $V_{\mathrm{mpp}}$.

\section{Discussion}

\subsection{Thermalization coefficient}

We first discuss the results which are not related to light trapping. The thermalization coefficient characterizes how strong the carriers will cool, hence the temperature increases with decreasing thermalization (Figs. 2b and $3 \mathrm{~b})$. The decrease in chemical potential is a consequence of the temperature change. When increasing the temperature just by changing the thermalization coefficient, the input and extracted current are only slightly affected. Therefore, the radiated power has to remain relatively constant (Eq. (7)). As the emission increases exponentially with the temperature (Eq. (10)), compensation occurs through a reduction of the chemical potential, so that $\mu / T$ remains constant within a term of order $\ln (T)$.

We want to comment on the typical thermalization factor values at which the transition between a conventional cell and zero thermalization cell occurs. We consider the absorber as a blackbody at temperature $T$ and chemical potential $\mu=0$, and we choose a black body of temperature $T_{S}=5800 \mathrm{~K}$ for the input power. By fixing the chemical potential, we remove the current balance (Eq. (7)), by assuming Auger process to dominate [20]. At open circuit, the equation to be solved becomes:

$$
\sigma\left(T_{S}^{4}-T^{4}\right)=Q\left(T-T_{L}\right) \exp \left[-\frac{E_{L O}}{k_{B} T}\right]
$$




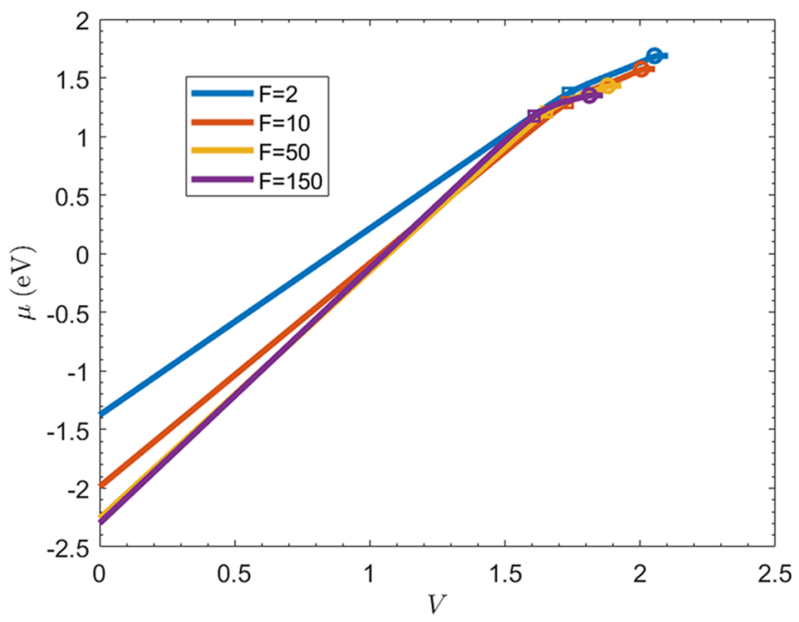

(a)

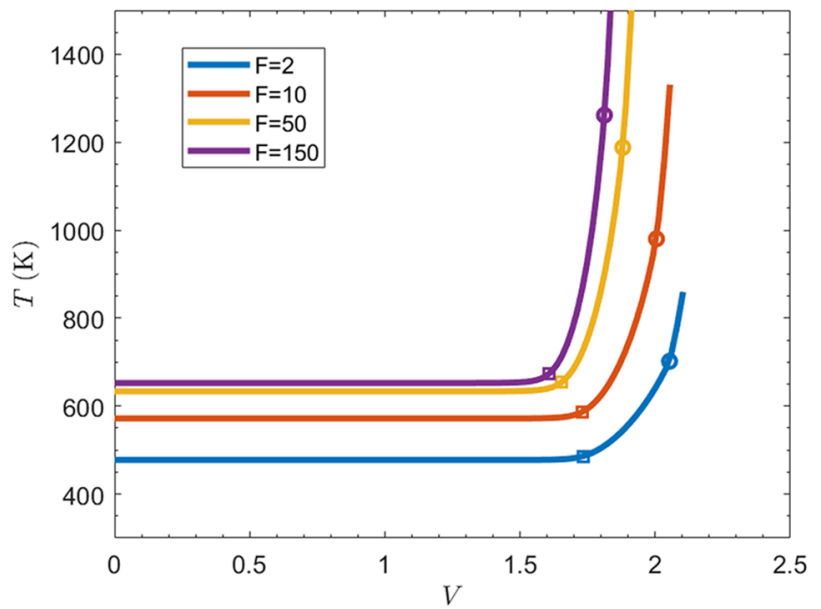

(b)

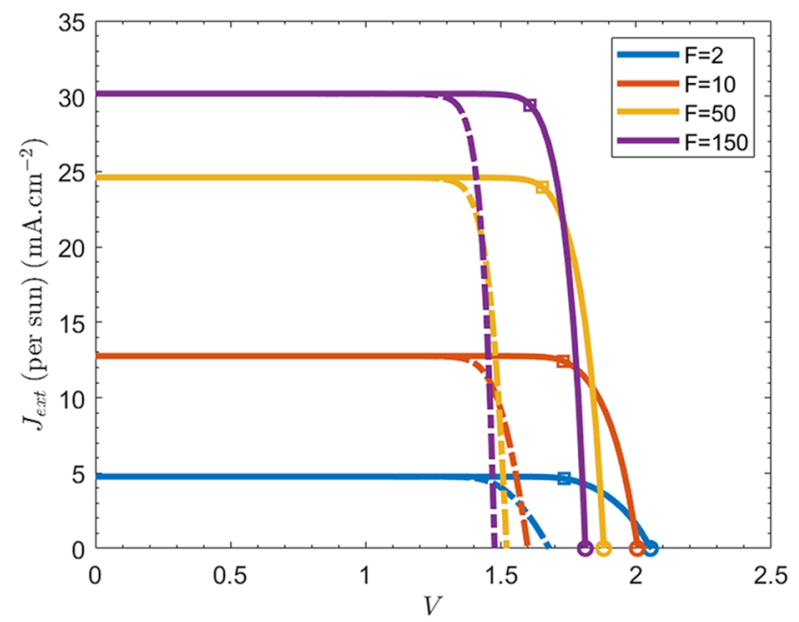

(c)

Fig. 4. (a) Chemical potential, (b) temperature of the carriers, and (c) extracted current (per sun) of a conventional cell and an HCSC, in dashed and continuous line, respectively, as a function of the applied voltage. The squares and circles respectively indicate the maximum power point and open circuit of the HCSC. These results are obtained for $Q=1 \mathrm{~W} \mathrm{~cm}^{-2} \mathrm{~K}^{-1}$.

where $\sigma$ is the Stefan-Boltzmann constant. This system admits for solutions $T=T_{S}$ for $Q=0$ and $T=T_{L}$ for $Q \rightarrow \infty$,

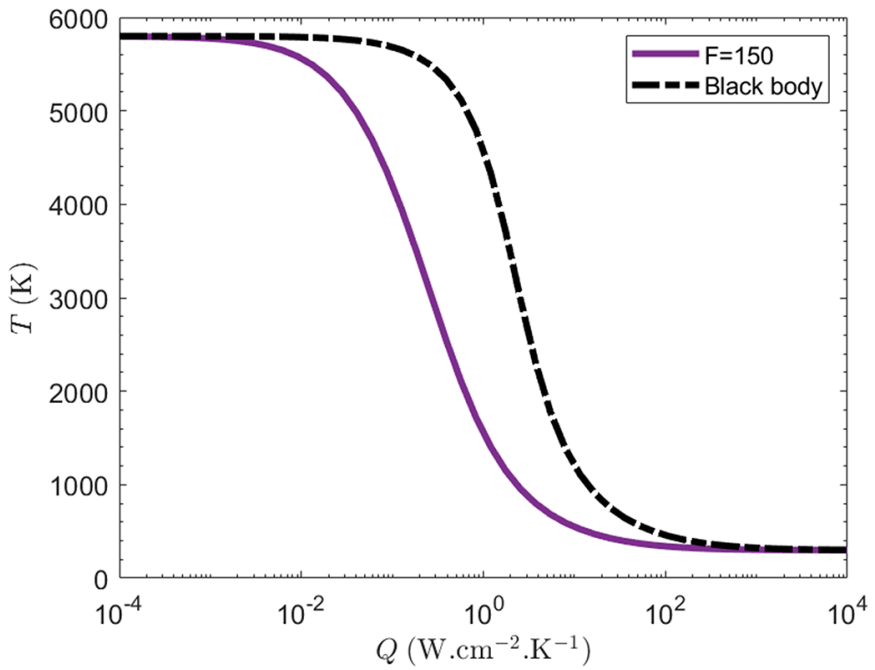

Fig. 5. Comparison of the evolution of the temperature in open circuit with the thermalization coefficient $Q$ in a black body and in the case $F=150$.

and so there must be a transition regime between those two extreme values. The solution of this equation as a function of $Q$ is plotted in Figure 5, along with the case $F=150$ (we consider for both a blackbody incident spectrum and the same value of $E_{\mathrm{LO}}$ ). The behavior of both curves is very similar so that equation (17) is what fundamentally governs the thermal excitation of carriers. We can see that the transition between a conventional cell and an HCSC occurs for $Q \in\left[10^{-1}, 10^{2}\right]$, which is higher and sharper than with highly absorbing GaAs. The curve for the blackbody can be thought as an upper limit for the temperature in the radiative limit (assuming $\mu \geq 0$ ). This means hot-carriers cannot appear in open circuit at room temperature for $Q \geq 100 \mathrm{~W}$ $\mathrm{cm}^{-2} \mathrm{~K}^{-1}$. We confirm the chemical potential is always positive for both GaAs (Fig. 2a) and GaSb (Supplementary Fig. S2a) in open-circuit.

Most of the difference between highly absorbing GaAs and a blackbody comes from the high bandgap of GaAs. This can be seen in Supplementary Figure S2b, where we show for comparison an absorber of GaSb with high absorptivity, but a bandgap twice as small. The temperature obtained in this material for a given thermalization factor is much higher and closer to the blackbody limit than for GaAs. Therefore, using materials with lower bandgap is also extremely important in generating hot-carriers.

It is interesting to compare the values required by our model with experimentally determined values of thermalization coefficient. We notice that values reported for room temperature in the literature are below $Q=$ $100 \mathrm{~W} \mathrm{~cm}^{-2} \mathrm{~K}^{-1}$ [2], as we have seen that higher values make it almost impossible to observe hot-carriers, even at full concentration. At room temperature, $Q=9.5 \mathrm{~W} \mathrm{~cm}^{-2} \mathrm{~K}^{-1}$ was measured in InGaAs-GaAsP quantum wells [5]. A similar value can be derived from the InGaAsP quantum well studied in [7]. These values are at the upper end of the spectrum in which we can hope to see a benefit from hotcarriers. It is therefore necessary to find ways to reduce this thermalization coefficient to reach values around $Q=1 \mathrm{~W} \mathrm{~cm}^{-2} \mathrm{~K}^{-1}$ and below. We also note that at low 
temperature, smaller values have been derived in InAsAlAsSb type-II quantum wells [21], with a thermalization coefficient as low as $Q=0.2 \mathrm{~W} \mathrm{~cm}^{-2} \mathrm{~K}^{-1}$ for a lattice temperature of $10 \mathrm{~K}$. At room temperature, they obtained hot-carriers with a temperature independent of the incident power, so that no thermalization coefficient can be derived.

\subsection{Influence of light trapping}

We now discuss the impact of the light trapping on HCSC. To do so, let us first describe the effect of light trapping in a conventional cell. The model we introduced is based on a wavelength-independent light trapping, where the absorption coefficient $\alpha$ is multiplied by a factor $F$. When the initial absorptivity is small, a first order approximation leads to $A \approx F \alpha d$. This is always the case near the bandgap, thus for the radiated power. However, if the absorptivity is already significant before light trapping (far from the bandgap), the gain from light trapping is small and tends toward 0 (see Fig. 1). Hence, wavelength-independent light trapping will contribute more to radiated power (where most of the radiation occurs near the bandgap) than to absorbed power (where the part near the bandgap only represents a small share of the total power). Therefore, as we can observe on the right of Figures $2 \mathrm{a}$ and $3 \mathrm{a}$, the chemical potential becomes smaller with increasing light trapping. Quantifying this chemical potential decrease is difficult, as it also affects the band filling, with a retroactive action on the population. Simulations are thus very useful in showing that the reduction is not negligible, amounting to several tens of $\mathrm{meV}$ in the case shown in Figure 2a.

This reduction of the chemical potential is observed because the absorptivity enhancement is proportionally higher for the radiation than for the emission. If we enhance homogeneously the absorptivity in a system with a step absorption:

$$
\left\{\begin{array}{l}
A=0 \text { if } E<E_{g} \\
A \in] 0,1] \text { if } E \geq E_{g},
\end{array}\right.
$$

the $V_{\text {OC }}$ remains identical (see Supplementary Fig. S3). We thus obtain a simple qualitative relation between the enhancement of the absorbed current and the chemical potential. If light trapping favors more the emission wavelengths than the absorption wavelengths, the chemical potential will be reduced, and conversely. The counterintuitive conclusion is that, with increasing light trapping, the density of carriers in the absorber in open circuit decreases due to enhanced emission.

The question is now to know whether this is still valid for a HCSC when reducing the thermalization coefficient. To understand this, we can compare the case of an absorber of thickness $d_{1}$ and light path enhancement $F_{1}$, to another with $d_{2}$ and $F_{2}>F_{1}$ such that $F_{1} d_{1}=F_{2} d_{2}$. The absorptivity of both systems is therefore identical. The expressions for the input and output current and power remain the same. In open-circuit, only the expression of the thermalization factor is affected [22], resulting in:

$$
Q_{2}=\frac{F_{2}}{F_{1}} Q_{1}
$$

With this comparison, changing light trapping results in a shifting of the $\mu$ and $T$ curves along the abscissa. This key result shows that light trapping enables the reduction of the thermalization through a reduction of the thickness, as long as the absorptivity remains constant. The case we show in Figure 2 is slightly different (the thicknesses are kept constant and only the light path is increased). Still, the same conclusion can be drawn as for a given $Q$, the temperature increases with $F$.

\subsection{Limit values of the chemical potential}

We want to discuss the peculiar results we observe for the chemical potential. In conventional cells, the chemical potential is limited between 0 and the absorber bandgap $E_{G}$. However, our results show chemical potentials both above and below these limits.

We will first discuss the case $\mu>E_{G}$, observed for example in Figures 2a and 4a. In Shockley-Queisser limit, the step absorber condition is equivalent to supposing an infinite density of states above the bandgap $\left(\alpha_{0}=\infty\right)$, and 0 below $\left(\alpha_{0}=0\right)$. This has for consequence that the chemical potential (identical to the voltage in conventional cells) cannot be higher than the bandgap, as this would cause $\alpha=-\infty$ (Eq. (5)), and infinite radiated power $\left(A(E)^{*} \Phi(E)=\infty\right.$ for $\left.E_{G}<E<\mu\right)$. Said otherwise, in the step absorber limit, the radiated current $J_{\text {rad }}$ increases as the chemical potential tends toward the bandgap, and reaches a singularity for $\mu \rightarrow E_{G}$.

Because we consider realistic finite absorption, the density of states progressively increases around the bandgap following an Urbach tail, and always remains finite (and so does $\alpha_{0}$ ). As such, the notion of bandgap is not as clear, and the chemical potential is no longer limited by the bulk bandgap value, especially for thin absorbers (see Fig. 2a for example). Of course, as the chemical potential increases above the bandgap, the emission becomes very high, and thus, this can only be observed for very high incoming light intensity. The argument developed in Section 4.2 also explains why the chemical potential reaches higher values for lower absorptivity.

This model limit is unlikely to be observed experimentally, as the presence of non-radiative defects should decrease the value of the bandgap very rapidly below the bandgap. Also, the Auger effect will have an important impact in this regime of high carrier density and will reduce the carrier density (in favor of a hotter population).

We now discuss the case $\mu<0$. We observe that the chemical potential of the cell can be negative for low thermalization factors, low concentration, and/or small applied voltage (Figs. 3a, 4a and S4a). The same result was also obtained by Ross and Nozik [1]. This is something impossible in forward-biased conventional cells, where the chemical potential is equal to the voltage (in an ideal device). However, in HCSC, these two parameters can be different, and are related through equation (14). In this equation, we can see that the voltage comes from two contributions. The first term contains the chemical potential, and corresponds to the operation of a conventional cell when $T=T_{L}$. It is the 
same sign as the chemical potential. The second term depends on the carrier temperature and the extraction energy $E_{\text {ext }}$. It is equal to zero in conventional cells, and is positive in HCSC. This second term can be seen as a thermoelectric conversion from a hot-absorber to a cold reservoir (a heat engine, in the terms of Ross and Nozik), to which we can associate a Seebeck coefficient [23]. Therefore, a positive voltage can be obtained with a negative chemical potential, as long as the carriers are hot enough.

From a thermodynamic perspective, the fact that $\mu$ becomes negative simply results from the conservation of the number of particles, fixed by the number of photons absorbed. By reducing the thermalization coefficient, we increase the temperature of the carriers (Eq. (12)), and because the particle number is constant, this results in a decrease in the chemical potential (Eqs. (3) and (4)). This can be observed when comparing Figures $2 \mathrm{a}$ and $2 \mathrm{~b}$, for example. The chemical potential is driven by the temperature (we can see that the temperature increase first before the chemical potential starts decreasing, as we reduce the thermalization coefficient) and stops decreasing when thermalization becomes negligible. There is no mechanism to keep the chemical potential positive, and $\mu=0$ is not a particular value.

We can wonder whether we could observe this behavior experimentally. In the same way Auger effect becomes important for a high chemical potential, impact ionization will become significant for high temperature and low chemical potential, in order to redistribute the high kinetic energy into more carriers [20]. Other effects present in real devices, including intervalley scattering or non-radiative recombinations, also make it more difficult to reach $\mu<0$ regime under forward bias.

\subsection{Voltage dependence}

Let us now analyze the evolution of the absorber characteristics with the voltage (Fig. 4). The $J(V)$ curve evolves similarly for the HCSC and the conventional cell. At the short-circuit, the emitted current and power are negligible (because the density of electrons in the conduction band is extremely small). This implies $J_{\mathrm{sc}} \approx J_{\mathrm{abs}}$ for both paradigms. For the HCSC, we can write the thermalized power under short-circuit operation:

$$
P_{\mathrm{th}, \mathrm{sc}} \approx P_{\mathrm{abs}}-E_{\mathrm{ext}} J_{\mathrm{abs}} .
$$

Solving this gives the equilibrium temperature reached when emission is negligible. The corresponding $\mu_{\mathrm{sc}}$ can be obtained by solving equation (14). While the short-circuit condition imposes $\mu=0$ in the conventional cell, the corresponding chemical potential in an HCSC is quite negative, proportionally to the carrier temperature. Then, as long as emitted power and band filling are negligible, the short circuit current and temperature remain the same (Figs. 4b and 4c), and the chemical potential increases linearly with the voltage, with a slope $T / T_{L}$ (Eq. (14)), almost up to $V_{\mathrm{mpp}}$ (Fig. 4a). At this point, the slope of the chemical potential tends toward 0 , while the temperature starts increasing, almost vertically (Fig. 4b). We observe evaporative cooling [24], which is a decrease of the carrier temperature under extraction and see in Figure $4 \mathrm{~b}$ that this temperature variation is strongest near the $V_{\text {OC }}$. This implies that even if we can generate hot-carriers in open circuit condition, the carriers extracted at maximum power point will be much colder.

\subsection{Influence of concentration and non-radiative case}

We chose to fix the light concentration at the theoretical limit, around 45000 suns, as it is the one that provides the highest efficiency. However, it is not currently technically achievable, and high concentration involves very high tracking system costs. Therefore, it is also interesting to look into the impact of concentration on the characteristics of the cell. We show in Supplementary Figure S4 how the chemical potential and temperature evolve as a function of the thermalization coefficient, for different concentration levels. The result which can seem the most surprising is that the temperature reached for each concentration is almost the same. It is, however, easier to analyze if we replace this result in the context of light trapping. Contrarily to light path enhancement, concentration increases the amount of light absorbed without modifying the emissivity. Therefore, the spectrum repartition of the absorbed photons is not modified, and the temperature should not change for $Q=0$. In the other hand, the amount of absorbed photons is increased with no change in the radiated flux expression. As we saw, this results in a reduction of the chemical potential. If we take two systems with concentration and chemical potentials $\left(C_{1}, \mu_{1}\right)$ an $\left(C_{2}, \mu_{2}\right)$, we can write the following relation within Boltzmann approximation:

$$
\mu_{2}-\mu_{1}=\frac{k T}{q} \ln \left(\frac{C_{2}}{C_{1}}\right) .
$$

The values obtained in the limits $Q \rightarrow 0$ and $Q \rightarrow \infty$ in Figure S4a are in accordance with equation (21). Also, the transition between low and high temperature will be translated by $C_{2} / C_{1}$, accompanied with a reduction of a chemical potential. We observe a crossing of the curves in Figure S4b for intermediate thermalization coefficient, because the chemical potential of the higher concentration will decrease first.

As a final remark, we note that it would be interesting to add non-radiative recombinations to this model [25]. As they are proportional to the volume of the absorber, they can be introduced in the same way as thermalization. The main idea is that light trapping will not only result in reducing the thermalization, but also the non-radiative losses.

\section{Conclusion}

A simple detailed balance model to investigate the influence of light trapping on the characteristics of HCSC was presented. We explained the reason why the chemical potential within our model can be negative or higher than the bandgap. The first key result is that at open circuit, the temperature of the radiation should increase with light trapping, and experiments are 
ongoing to confirm this effect. Also, a strong variation of the carrier temperature near the open-circuit voltage was observed, confirming the idea of evaporative cooling. Furthermore, the key relationship between broadband absorption enhancement and the modification of the chemical potential was highlighted. Finally, the crucial role of light trapping to bring together high absorptivity and low thermalization coefficient was demonstrated. On one hand, low thermalization in quantum structures with low absorptivity cannot provide HCSC efficiencies anywhere close to conventional cells. On the other hand, no matter how good the absorption, hot-carriers at open circuit only start appearing for a thermalization coefficient $Q \leq 100 \mathrm{~W} \mathrm{~cm}^{-2} \mathrm{~K}^{-1}$, so low thermalization designs are necessary. The ultimate goal is to combine the best knowledge in HCSC absorbers and in nanophotonics in order to demonstrate cells operating under broadband concentrated light, with an efficiency gain as compared with conventional cells.

\section{Supplementary material}

Figure S1 Comparison of the multi-resonant absorption with the step absorber characteristics as a function of the applied voltage. The case $F=150$ is also shown in Figure 4. Figure S2 (a) Comparison of the evolution of the temperature in open circuit with the thermalization coefficient $Q$ in a black body and in a 10 nm-thick GaSb absorber with $F=150$. (b) Corresponding chemical potential of the GaSb absorber.

Figure S3 Extracted current (per sun) as a function of the applied voltage for a thermalized GaAs cell in the radiative limit for different values of homogeneous absorptivity. The open circuit voltage is the same for each case.

Figure S4 (a) Chemical potential and (b) temperature of the radiation in open-circuit condition as a function of the thermalization coefficient $Q$, for different concentrations. The results are obtained for $F=150$. The case $C=45000$ is also shown in Figure 2 (different scale).

Table S1 Parameters of conventional cells and HCSC obtained from the detailed balance calculation with $Q=1 \mathrm{~W} \mathrm{~cm}^{-2} \mathrm{~K}^{-1}$.

The Supplementary Material is available at https://www. epj-pv.org/10.1051/epjpv/2019001/olm.

This work is supported by the Incorporated Administrative Agency New Energy and Industrial Technology Development Organization (NEDO) and the Ministry of Economy, Trade and Industry (METI), Japan.

\section{Author contribution statement}

The code and article were written by Maxime Giteau. Daniel Suchet, Jean-François Guillemoles, and Stéphane Collin brought valuable answers to difficult aspects of this work, and critical feedback all along the development of the research. Yoshitaka Okada provided general supervision of Maxime Giteau's work.

\section{References}

1. R.T. Ross, A.J. Nozik, J. Appl. Phys. 53, 3813 (1982)

2. A.L. Bris et al., Energy Environ. Sci. 5, 6225 (2012)

3. Y. Rosenwaks et al., Phys. Rev. B 48, 14675 (1993)

4. J. Tang et al., Appl. Phys. Lett. 106, 061902 (2015)

5. L.C. Hirst, H. Fujii, Y. Wang, M. Sugiyama, N.J. EkinsDaukes, IEEE J. Photovolt. 4, 244 (2014)

6. L.C. Hirst, R.J. Walters, M.F. Führer, N.J. Ekins-Daukes, Appl. Phys. Lett. 104, 231115 (2014)

7. D.-T. Nguyen et al., Nat. Energy 3, 236 (2018)

8. E. Yablonovitch, J. Opt. Soc. Am. 72, 899 (1982)

9. S. Collin, M. Giteau, in Proceedings of the World Conference on Photovoltaic Energy Conversion (WCPEC-7), 2018

10. F. Gibelli, L. Lombez, J.-F. Guillemoles, Physica B 498, 7 (2016)

11. V.R. Whiteside et al., Semicond. Sci. Technol. 34, 025005 (2019)

12. W. Shockley, H.J. Queisser, J. Appl. Phys. 32, 510 (1961)

13. P. Wurfel, J. Phys. C: Solid State Phys. 15, 3967 (1982)

14. A.L. Bris, J.-F. Guillemoles, Appl. Phys. Lett. 97, 113506 (2010)

15. S.A. Lyon, J. Lumin. 35, 121 (1986)

16. A. Le Bris, $\mathrm{PhD}$ thesis, Châtenay-Malabry, Ecole Centrale de Paris, 2011

17. S.C. Limpert, S.P. Bremner, Appl. Phys. Lett. 107, 073902 (2015)

18. M.A. Green, Prog. Photovolt.: Res. Appl. 20, 472 (2012)

19. E.D. Palik, in Handbook of Optical Constants of Solids, edited by E.D. Palik (Academic Press, Burlington, 1997)

20. P. Würfel, A.S. Brown, T.E. Humphrey, M.A. Green, Prog. Photovolt: Res. Appl. 13, 277 (2005)

21. H. Esmaielpour et al., Prog. Photovolt.: Res. Appl. 24, 591 (2016)

22. M. Giteau et al., in Proceedings of the World Conference on Photovoltaic Energy Conversion (WCPEC-7), 2018

23. J. Rodière, L. Lombez, A. Le Corre, O. Durand, J.-F. Guillemoles, Appl. Phys. Lett. 106, 183901 (2015)

24. D. Suchet, Z. Jehl, Y. Okada, J.-F. Guillemoles, Phys. Rev. Appl. 8, 034030 (2017)

25. F.R.J. Gibelli, PhD thesis, Paris 6, 2016

Cite this article as: Maxime Giteau, Daniel Suchet, Stéphane Collin, Jean-François Guillemoles, Yoshitaka Okada, Detailed balance calculations for hot-carrier solar cells: coupling high absorptivity with low thermalization through light trapping, EPJ Photovoltaics 10, $1(2019)$ 